\title{
Elektronische Faksimile-Editionen von ausgewählten Werken der Fachbereichsbibliothek Astronomie an der Universitätssternwarte Wien
}

\author{
Hanns Petsch ${ }^{1}$ und Adrian Partl ${ }^{2}$ \\ ${ }^{1}$ Institut für Astronomie der Universität Wien, \\ Türkenschanzstraße 17, A-1180 Wien, Austria \\ 2 Astrophysikalisches Institut Potsdam, \\ An der Sternwarte 16, D-14482 Potsdam, Germany
}

\begin{abstract}
The Vienna University Observatory holds important collections of historically significant books in the field of astronomy and related disciplines. The library contains a total of 500 books printed prior to 1800 . We are trying to follow the tradition, that knowledge should be accessible for each and everybody. So we founded this project to digitally conserve these rare books and open them to the public.
\end{abstract}

\section{Einleitung}

Die Bibliothek an der Universitätssternwarte Wien bietet einen überaus vielfältigen und großen Schatz an alten Büchern zum Thema Astronomie, Mathematik und Physik. Ganz in der Tradition, dass Wissen jedem zugänglich sein sollte, wurde die Digitalisierung des Buchbestandes mit dem Ziel in Angriff genommen, die Bücher einem breiten Publikum verfügbar zu machen.

Die Bücher sind dadurch nicht nur jederzeit und allerorts zugänglich, auch werden so die Bücher vor einem erhöhten Gebrauch geschützt und konserviert. Das Projekt wurde von engagierten Studenten ins Leben gerufen und ehrenamtlich durschgeführt.

Die digitalen Faksimileeditionen dieser Bücher befinden sich auf der Homepage des Projektes unter http://www.univie.ac.at/hwastro in mehreren Qualitätsstufen zum kostenlosen Download bereit. 


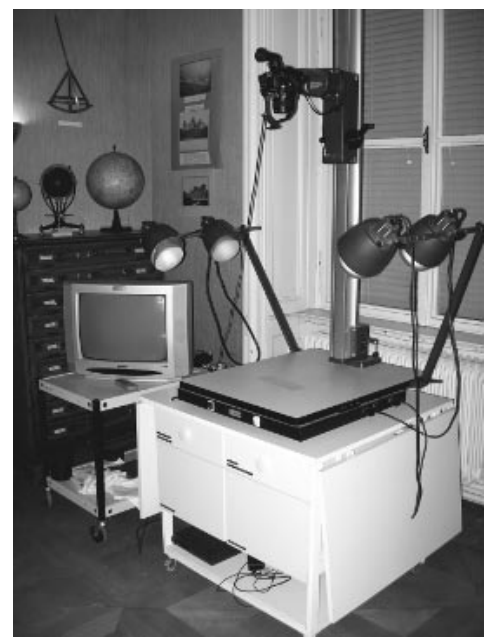

Abb. 1: Reproanlage

\section{Methodik - Ausrüstung}

Das Institut für Astronomie stellt uns freundlicherweise eine einfache aber zweckmäßige Reproanlage zur Verfügung. Die Anlage selbst besteht aus einer handelsüblichen Nikon D70 Digitalkamera mit 6 Mpixel Auflösung und einem verzerrungsfreien Makro-Objektiv (AF MICRO NIKKOR 60mm 1:2.8D). Eine sehr stabiles höhenverstellbares Stativ verbindet die Kamera mit dem ReproTisch, welcher über 4 Stk. Claudfar 150W Lampen homogen ausgeleuchtet wird. Als zusätzliches Hilfe wird ein Kontrollmonitor verwendet, um direkt nach dem Ablichten die Schärfe, Ausrichtung und Helligkeit zu überprüfen (siehe Abb. 1).

\section{Methodik - Digitalisierung}

Zuerst erfolgt ein Weißabgleich mittels geeichter Graukarte. Dadurch wird einerseits die Farbechtheit der Buchseiten garantiert und andererseits der dynamische Bereich der Kamera optimal ausgenützt. Das ausgewählte Werk wird dann parallel zu den Kanten der Kamera ausgerichtet und der Bereich des Buches auf dem Repro-Tisch gekennzeichnet, um spätere Verschiebungen korrigieren zu können. Eine möglichst konstante Ausrichtung ist unabdingbar, um die nachfolgende digitale Bildbearbeitung effizient durchführen zu können, deshalb werden erst alle rechten Seiten des Buches und danach alle linken abgelichtet. 
Die oben beschriebene Methode mag zwar angesichts der heutigen technischen Möglichkeiten wie Buchscannern etc. veraltet und langsam erscheinen, bietet dafür aber eine sehr geringe Beanspruchung der Bücher, was für uns im Vordergrund steht. Zusätzlich dazu kann die Digitalisierung direkt in der Bibliothek erfolgen, es entfallen daher Transportwege und logistische Probleme wie Verpackung und Versicherung.

\section{Methodik - Verarbeitung}

Die digitalen Rohdaten werden dann teilweise von Hand, teilweise automatisiert verarbeitet. Die ersten Schritte der Handbearbeitung beinhalten das Drehen der Bilder und Retouchieren jeglicher Halte-Hilfsmittel. Wenn nötig, werden die Seiten zentriert, sodass alle Buchseiten übereinander liegen. Nach diesen Schritten können die Bilder automatisch verarbeitet werden. Zuerst werden die Seiten auf eine einheitliche Größe zugeschnitten. Dann erfolgt die Verringerung der Qualität in unsere zwei Qualitätsstufen Mittel und Niedrig, sowie die Umwandlung von Farbe in Schwarz-Weiß.

Sind diese Schritte ausgeführt, fügt ein Skript mittels der in der Astronomie weit verbreiteten Setzersprache LaTeX die Bilder zu einem PDF-File zusammen. Sofern vorhanden werden noch Zusatzinformationen zu den einzelnen Büchern den PDF-Dateien beigefügt und dann im Internet veröffentlicht. Die Faksimile Ausgaben sind in den Varianten Farbe und Schwarz-Weiß erhältlich. Diese sind jeweils in den beiden Qualitätsabstufungen Mittel und Niedrig ausgeführt.

Die mittlere Qualitätsabstufung ist so gewählt, dass alle Details erkennbar sind. Die niedrige Qualität hingegen ermöglicht nur das Lesen der Schrift, aber nicht mehr das Erkennen der kleinsten Details.

\section{Veröffentlichungen}

Ende des Jahres 2007 stehen bereits 13 Bücher digital im Internet zu Verfügung. Dabei wurde die Sicherung des 15. Jahrhundert komplett abgeschlossen. Insgesammt sind dies folgende Bücher:

1473: Peuerbach: Theoricae novae planetarum

1476: Regiomontanus: Calendarium

1496: Regiomontanus: Epytoma in Almagestum Ptolemaei (siehe Abb. 2 links)

1498: Nicephorus: Logica (siehe Abb. 2 rechts)

1499: Pseudo Proclus Diadochus: Sphaera

1505: Heinrich v. Langenstein: Secreta sacerdotum qu[a]e in missa teneri debent

1515: Claudius Ptolemaeus: Almagestum

1517: Jacques Lefèvre d'Etaples: Introductorium astronomicum

1540: Petrus Apian: Astronomicum caesareum (siehe Abb. 3 links) 
1606: Johannes Kepler: De Stella nova

1662: Johannes Hevelius: Mercurius in Sole visus Gedani

1770: Maximilian Hell: Observatio transitus Veneris antediscum Solis

die 3 Junii anno 1769

1793: Maximilian Hell: Beobachtung des Durchganges der Venus durch die Sonnenscheibe (siehe Abb. 3 rechts)

Wir können auf der Projektseite einen konstanten Besucherstrom von durchschnittlich 10 Besuchern täglich verzeichnen. Bemerkenswert dabei ist, dass die Zugriffe von den unterschiedlichsten Ländern der Welt stammen. Auch die Vernetzung mit wichtigen Internet-Seiten wie Google und Wikipedia führt Interessierte auf unser Projekt.

\section{Ausblick}

Wir werden die unentgeltliche Arbeit unter Rücksichtnahme auf die Fachgemeinschaft weiterführen und zusätzlich die uns interessant erscheinenden Bücher digitalisieren. Wir hoffen dadurch die Forschungsarbeit zu erleichtern.

Wir danken F. Kerschbaum und Th. Posch für ihre Schirmherrschaft und tatkräftige Unterstützung. Ohne sie währe dieses Projekt nicht durchführbar. Weiters danken wir U. Kuchner für ihre Hilfe bei der Digitalisierung und R. Partl für die Übersetzung der einleitenden Texte ins Englische. 

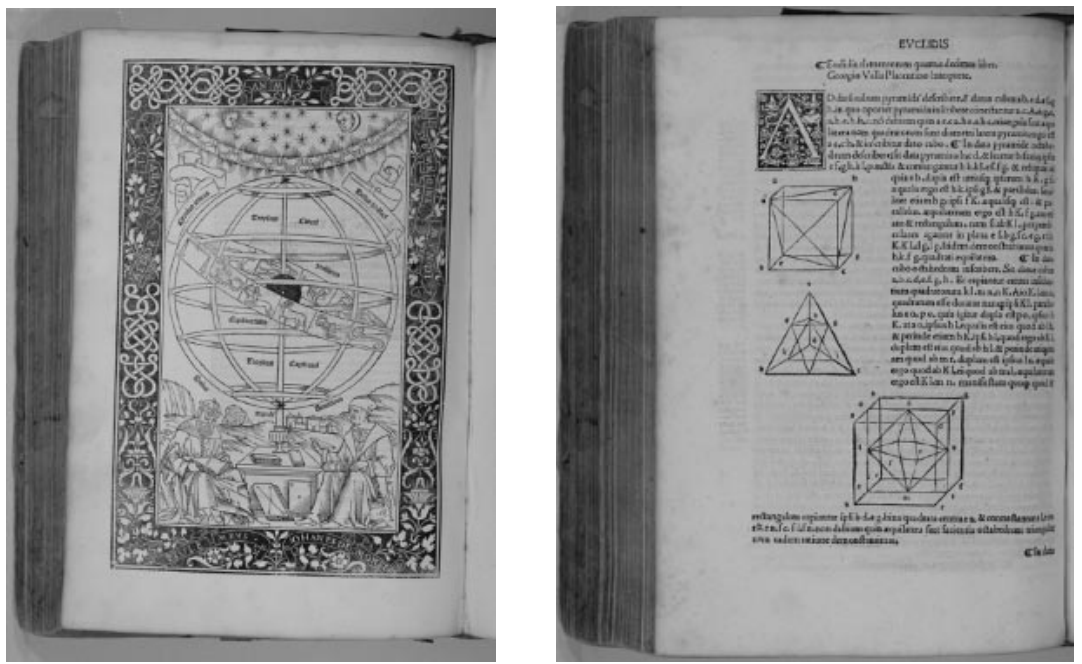

Abb. 2: Regiomontanus, 1496, p. 6 bzw. Nicephorus, 1498, p. 46
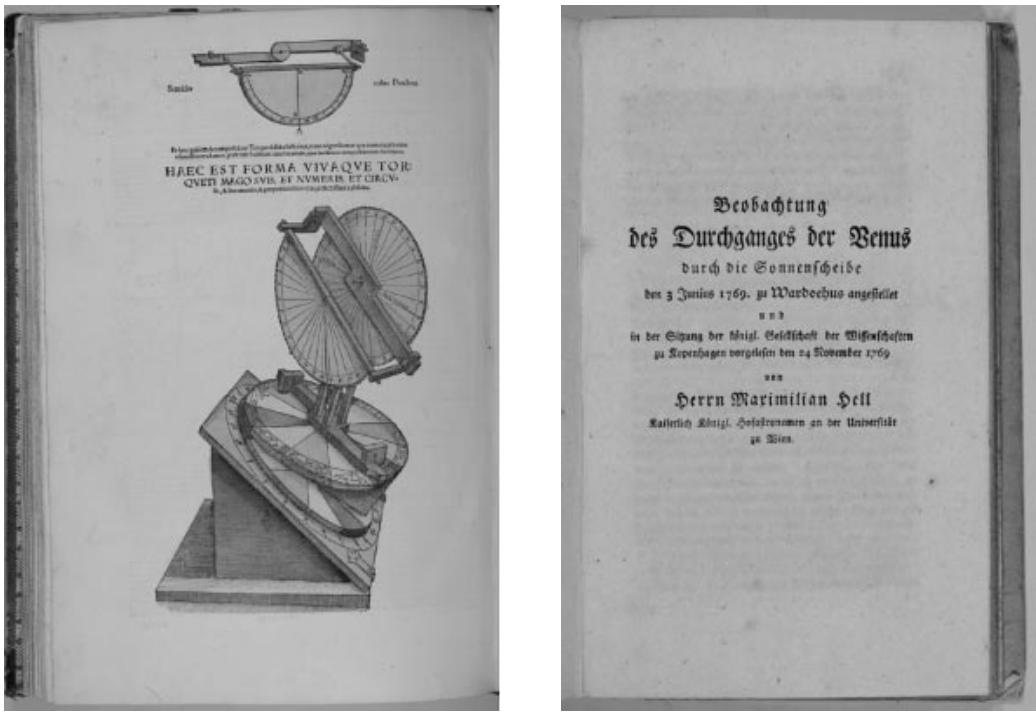

Abb. 3: Apian, 1540, p. 119 bzw. Hell, 1793, p. 18 